\begin{tabular}{l|c|c|c|}
\hline Bentham open & The Open Dentistry Journal \\
CrossMark & Content list available at: www.benthamopen.com/TODENTJ/ \\
DOI: $10.2174 / 1874210601610010207$ & $\begin{array}{c}\text { The } \\
\text { Open Dentisty } \\
\text { lournal }\end{array}$ \\
\hline
\end{tabular}

\title{
Comparison of Dill Seed Oil Mouth Rinse and Chlorhexidine Mouth Rinse on Plaque Levels and Gingivitis - A Double Blind Randomized Clinical Trial
}

\author{
Shruthi Eshwar ${ }^{1, *}$, Rekha K ${ }^{1}$, Vipin Jain ${ }^{1}$, Supriya Manvi ${ }^{2}$, Shivani Kohli ${ }^{3}$ and Shekhar Bhatia ${ }^{4}$ \\ ${ }^{I}$ Department Public Health Dentistry, KLES Institute of Dental Sciences, Bangalore-22, India \\ ${ }^{2}$ Department Prosthodontics, KLES Institute of Dental Sciences, Bangalore-22, India \\ ${ }^{3}$ Department of Prosthodontics, MAHSA University, Kuala Lumpur, Malaysia \\ ${ }^{4}$ Division of Restorative Dentistry, School of Dentistry, International Medical University, Kuala Lumpur, Malaysia
}

Received: December 30, 2015

Revised: January 1, 2016

Accepted: January 28, 2016

\begin{abstract}
:
Introduction:

Mouthrinses have been in use for centuries as breath fresheners, medicaments, and antiseptics. Dill is said to be a good source of calcium, manganese and iron. It contains flavonoids known for their antioxidant, anti-inflammatory, and antiviral properties. Dill can help with microbial infections in the mouth; and its anti-oxidants minimize damage caused by free radicals to the gums and teeth. Being a good source of calcium, dill also helps with bone and dental health.
\end{abstract}

\section{Aims and Objectives:}

To compare the effectiveness of commercially available $0.2 \%$ chlorhexidine gluconate mouthrinse and dill seed oil mouthrinse on plaque levels and gingivitis.

\section{Material and Methods:}

A randomized controlled, double blind parallel arm study was conducted over 90 days on 90 subjects. The subjects were randomly divided into 2 groups and baseline data was collected using Loe and Silness gingival index and Quigley Hein plaque index and oral prophylaxis was performed on all the subjects. The mouthrinses included in the present study were dill seed oil and Hexodent $(0.2 \%$ chlorhexidine gluconate). Intervention regarding the mouthrinsing was given to the subjects and were followed up for 45 days and 90 days, after this post intervention changes were assessed using the respective indices.

\section{Results:}

It was observed that there is no significant difference in gingival \& plaque scores among two mouthrinses from baseline to 45 days and 90 days. It was observed that there is statistical difference in gingival and plaque scores when compared with baseline to 45 days $(p<0.001)$, baseline to 90 days $(p<0.001)$ and 45 days to 90 days $(p<0.001)$ when intergroup comparisons were done.

\section{Conclusion:}

It was concluded that dill seed oil and Hexodent ( $0.2 \%$ chlorhexidine gluconate) mouthrinse have similar antiplaque and antigingival effectiveness.

Keywords: Chlorhexidine gluconate, dill seed oil, gluconate, gingivitis, plaque.

\footnotetext{
* Address correspondence to this author at the Department Public Health Dentistry, KLES Institute of Dental Sciences, Bangalore-22, India; E-mail: shruthy2015@gmail.com
} 


\section{INTRODUCTION}

The oral cavity provides a unique ecosystem in the human body. Its moist environment, temperature, and the existence of endogenous and exogenous metabolic substrates make it an ideal medium for bacterial growth. It is widely accepted in dentistry that plaque containing a combination of pathogenic micro-organisms is a principal etiological factor associated with periodontal disease [1].

The incorporation of broad spectrum antimicrobial mouthrinses as adjuncts to patients daily oral hygiene regimens has assumed greater importance with the recognition that most individuals are unable to consistently maintain adequate levels of plaque control using mechanical methods alone [2].

The finding that a $0.2 \%$ chlorhexidine mouthrinse can effectively prevent plaque and gingivitis in the absence of other oral hygiene procedures using an experimental gingivitis study model established the potential for antimicrobial mouthrinses in clinical practice [3]. However, because of certain side effects associated with this agent, in particular tooth staining, calculus formation, and taste aberrations, it was deemed desirable to be used as medicated rinses [4].

Dill (Anethum graveolens), also known as Shapt or dill-weed, belongs to family Umbelliferae, same family as parsley, cumin and bay leaf. It has been used as a medicine and a spice since ancient times by both the Greeks and the Romans. The main constituents of dill oil which is pale yellow in color, darkens on keeping, with the odor of the fruit and a hot, acrid taste is a mixture of a paraffin hydrocarbon and 40 to $60 \%$ of d-carvone $(23.1 \%)$ with d-limonene (45\%). It also consists of $\alpha$-phellandrene, eugenol, anethole, flavonoids, coumarins, triterpenes, phenolic acids and umbelliferone [5].

Dill is said to be a good source of calcium, manganese and iron. It contains flavonoids known for their antioxidant, anti inflammatory and antiviral properties. The health benefits of dill include good digestion, relief from insomnia, hiccups, diarrhea, dysentery, menstrual disorders, respiratory disorders, cancer, to name a few. But what is unaware to the public is that dill is beneficial for oral care as it helps with bone and oral health. It also acts as an anti microbial agent.

Development of bacterial resistance to synthetic antimicrobial agents and the side effects associated with their use favour essential oils for alternative or complementary use. In India, antimicrobial resistance has been reported in for the most predominant pathogenic microorganisms including Staphylococcus aureus, Enterococcus faecalis, Mycobacterium tuberculosis and Pseudomonas. aeruginosa. Since dill seed oil has proven anti microbial properties with no tendency for microbial resistance, its ease of availability and relative cost it can be used as a alternate mouth rinse. However, not much research work has been done comparing the effect of dill seed oil mouthrinses and chlorhexidine towards dental plaque and gingivitis in controlled clinical trials [5].

So, this study attempts to assess the anti-plaque and anti-gingival effectiveness of commercially available $0.2 \%$ chlorhexidine gluconate mouthrinse and dill seed oil mouthrinse.

\section{MATERIALS AND METHODS}

The present study was a randomized double blind parallel arm clinical study with an aim to compare the effectiveness of commercially available $0.2 \%$ chlorhexidine gluconate mouth rinse and dill seed oil mouth rinse on plaque levels and gingivitis of study subjects. The study was conducted among patients who gave consent and met the selection criteria over a period of 3 months in Department of public health dentistry, KLE Society's Institute of Dental Sciences, Bangalore. Ethical clearance for the study was taken from the Ethical clearance committee.

\section{Inclusion Criteria}

- Subjects with a minimum of 20 sound natural teeth.

- Subjects with gingivitis.

- Subjects available for entire duration of the study.

- Subjects aged between 20 - 40 years.

\section{Exclusion Criteria}

- Subjects with severe malocclusion / Orthodontic appliance or more than one tooth with prosthetic crown.

- Subjects who required immediate health care / Destructive periodontal disease. 
- Subjects undergoing antibiotic, steroid therapy or any anti-inflammatory drugs in the preceding month.

- Subjects with history of allergies to dental products or their ingredients.

- Medically compromised patients.

A total of 90 subjects were selected for the study. Group sample size of 45 and 45 to achieve $80 \%$ power to detect difference of 0.21 (marginal error) between the experimental drug and the control. The group means assumed 1.04 (0.003), 1.51 (0.003) in experimental group and control group respectively, with the significance level of 0.05 using two sided two sample $t$-test. Randomization was done by using computer generated table of random numbers.

Group A ( $\mathrm{n}=45)$ - rinsed with dill seed oil mouth rinse

Group B ( $\mathrm{n}=45)$ - rinsed with $0.2 \%$ Chlorhexidine gluconate

The Kappa co-efficient value (k) for intra examiner reliability for the investigator was 0.89 for plaque index and 0.88 for gingival index. The overall intra-examiner reliability with respect to the gingival and plaque index was good.

\section{MANUFACTURING OF DILL SEED OIL BASED MOUTHRINSE}

Glycerin, Propylene glycol and Tween 80 (Excipients) in required quantity are mixed in a clean vessel. Add Dill Seed Oil slowly and stir continuously till it mixes well. Then sufficient quantity of demineralised (DM) water is added to adjust the volume and stir well. Finally the colour is added and mix thoroughly for a homogeneous liquid. This liquid is used as mouth rinse.

\section{Study Procedure}

On day 1 of study period, all the subjects underwent an oral soft and hard tissue examination and a professional scaling and polishing to remove calculus, plaque and extrinsic tooth stain to ensure that all deposits were removed. The subjects in the group A received a dill seed oil mouthrinse, whereas subjects in the group B received $0.2 \%$ chlohexidine mouthrinse. All participants were instructed to refrain from using any other means of oral hygiene during the experimental period. All subjects were instructed to rinse twice a day in morning and in evening with $20 \mathrm{ml}$ solution for 60 seconds. The subjects were instructed not to rinse with water immediately. Written instructions were provided explaining how to use the mouthwash. Rinsing was performed at home without supervision. To check for compliance, subjects were asked to note the time of day when they rinsed. After 45 and 90 days, erythrosine solution was used to assess plaque levels and gingivitis in both groups using the Quigley and Hein Plaque index as modifiedby Turesky et al. and Loe and Sillness gingival index respectively. All measurements were carried out under the same conditions by the same blinded investigator (Fig. 1).

\section{STATISTICAL ANALYSIS}

- The data collected were entered into excel spread sheet. The univariate and bivariate tables were generated using the statistical software SPSS (Version 13.0).

- The descriptive statistics for gingival and plaque index scores were computed.

- The comparision of dill seed oil and chlorhexidine groups were made using paired $t$-test.

\section{RESULTS}

The total mean scores of the gingivitis were compared at 45 days and 90 days. In both the groups there was a significant reduction of gingival scores from baseline to 45 days and from 45 days to 90 days.

In dill seed oil group the total mean baseline score was 1.75 , at 45 days it reduced to 1.14 and at 90 days it was 0.75 . In chlorhexidine group the total mean baseline score was 1.77 , at 45 days it was 1.18 and at 90 days it was reduced to 0.70 . This shows that the dill seed oil and chlorhexidine mouthrinse had demonstrable effect on gingivitis at 45 and 90 days (Fig. 2).

The total mean scores of the plaque index were compared at 45 days and 90 days. In both the groups there was a reduction of plaque scores from baseline to 45 days and from 45 days to 90 days. Dill seed oil group the total mean baseline score was 2.69 , at 45 days it reduced to 1.91 and at 90 days it reduced to 1.32 . In chlorhexidine group the total mean baseline score was 2.88 , at 45 days it reduced to 1.87 and at 90 days it was reduced to 1.11 . This shows that the dill seed oil and chlorhexidine mouthrinse had demonstrable effect on plaque at 45 and 90 days (Fig. 3). 


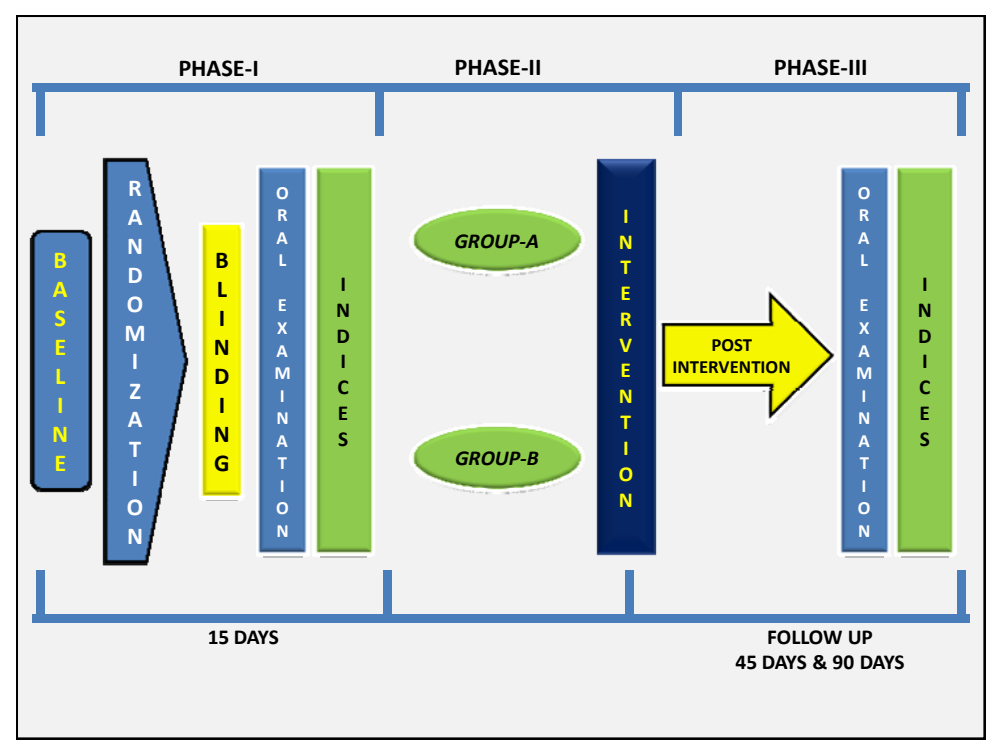

Fig. (1). Diagrammatic representation of study design.

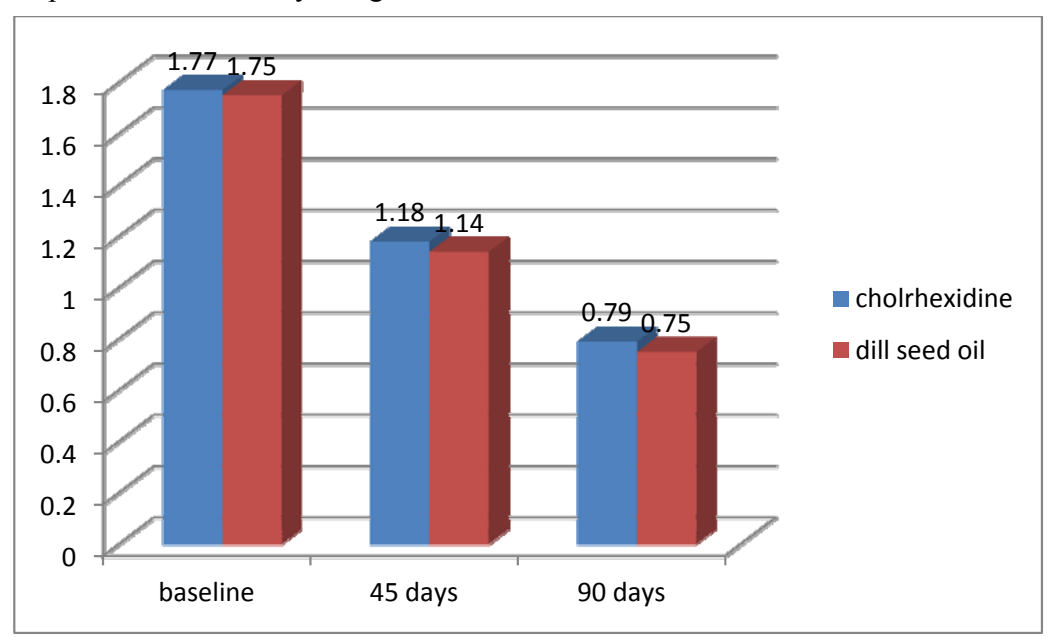

Fig. (2). Synthesis of Mannich bases of Pyrazole-5-one phosphonates 6(a-h).

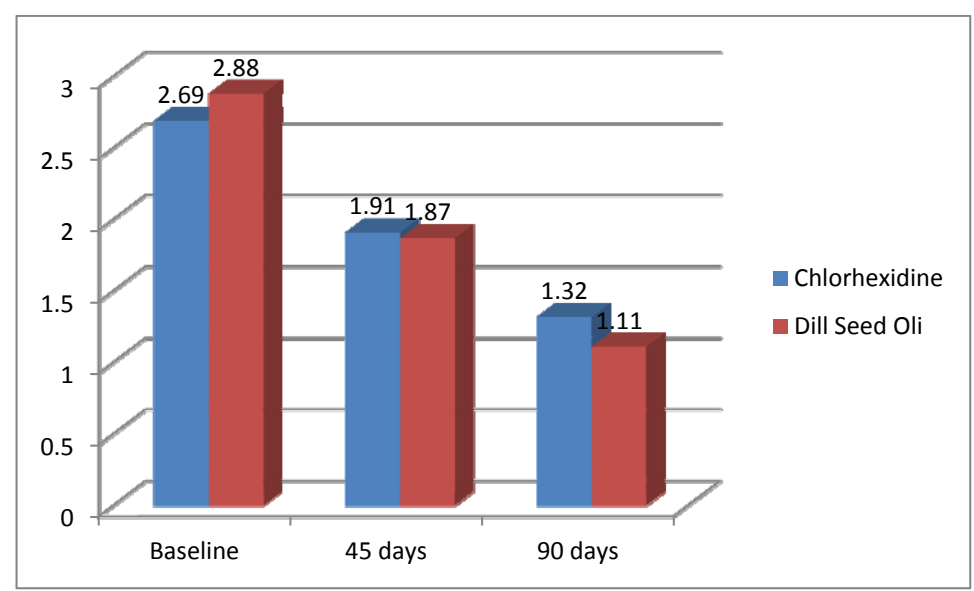

Fig. (3). Distribution of mean Plaque scores across the groups.

When dill seed oil was compared with chlorhexidine group, there was no statistically significant difference in gingival index scores at both 45 days $(p=0.69)$ and 90 days $(p=0.76)$. It shows that there is no significant difference in gingival scores from baseline to 45 and 90 days between essential oil and chlorhexidine group (Table 1). 
When dill seed oil was compared with chlorhexidine group, there was no statistically significant difference in plaque index scores at both 45 days $(p=0.62)$ and 90 days $(p=0.79)$. It shows that there is no significant difference in gingival scores from baseline to 45 and 90 days between essential oil and chlorhexidine group (Table 2).

It was observed that there is statistical difference in gingival and plaque scores when compared with baseline to 45 days $(p<0.001)$, baseline to 90 days $(p<0.001)$ and 45 days to 90 days $(p<0.001)$ in both dill seed oil group and in chlorhexidine group (Tables 3, 4).

Table 1. Comparision of gingival index scores between dill seed oil and chlorhexidine group.

\begin{tabular}{|c|c|c|c|c|c|}
\hline \multicolumn{2}{|c|}{ Baseline } & \multicolumn{2}{c|}{ 45 days } & \multicolumn{2}{c|}{$\mathbf{9 0}$ days } \\
\hline$t$-value & $p$-value & $t$-value & $p$-value & $t$-value & $p$-value \\
\hline 0.22 & 0.59 & 0.50 & 0.69 & 0.72 & 0.76 \\
\hline
\end{tabular}

Table 2. Comparision of plaque index scores between dill seed oil and chlorhexidine group.

\begin{tabular}{|c|c|c|c|c|c|}
\hline \multicolumn{2}{|c|}{ Baseline } & \multicolumn{2}{c|}{ 45 days } & \multicolumn{2}{c|}{90 days } \\
\hline$t$-value & $p$-value & $t$-value & $p$-value & $t$-value \\
\hline 1.29 & 0.90 & 0.33 & 0.62 & 2.62 \\
\hline
\end{tabular}

Table 3. Intragroup comparision of means between various intervals - dill seed oil group.

\begin{tabular}{|c|c|c|c|c|}
\hline \multirow[t]{2}{*}{ Variables } & \multicolumn{2}{|c|}{ Gingival Scores } & \multicolumn{2}{|c|}{ Plaque Scores } \\
\hline & Paired $t$ & $p$-value & Paired $t$ & $p$-value \\
\hline Baseline $v s 45$ days & 17.29 & $p<0.001^{*}$ & 13.95 & $p<0.001^{*}$ \\
\hline 45 days $v s 90$ days & 15.81 & $p<0.001^{*}$ & 17.34 & $p<0.001^{*}$ \\
\hline Baseline vs 90 days & 22.26 & $p<0.001^{*}$ & 20.57 & $p<0.001^{*}$ \\
\hline
\end{tabular}

(*p value significant)

Table 4. Comparision of plaque index scores between dill seed oil and chlorhexidine group.

\begin{tabular}{|c|c|c|c|c|}
\hline Variables & \multicolumn{2}{|c|}{ Gingival Scores } & \multicolumn{2}{c|}{ Plaque Scores } \\
\hline & Paired $t$ & $p$-value & Paired $t$ & 17.85 \\
\hline Baseline $v$-value 45 days & 18.06 & $p<0.001^{*}$ & $p<0.001^{*}$ \\
\hline 45 days $v$ s 90 days & 10.58 & $p<0.001^{*}$ & 11.76 & $p<0.001^{*}$ \\
\hline Baseline $v$ 9 90 days & 22.96 & $p<0.001^{*}$ & 21.63 \\
\hline
\end{tabular}

(*p value significant)

\section{DISCUSSION}

Plaque is the primary etiologic agent in the development of dental caries, gingivitis and periodontal diseases. Mechanical removal of plaque through frequent and efficacious brushing and flossing is the principal means of preventing periodontal diseases and diminishing the risk of oral diseases. Apart from the physically challenged and medically compromised individuals, even normal individuals might not be able to perform optimal mechanical cleansing. Additional means of plaque control in the form of mouthrinses thereby becomes an important adjunct in maintaining optimal oral hygiene [6].

Mouthrinses have been used for centuries for medicinal and cosmetic purposes, but it is only in recent years that the rationale behind the use of ingredients has been subjected to scientific research and clinical trials. The advent of mouth rinses containing chlorhexidine has been a major breakthrough in the research as a chemical means to prevent oral diseases. Since its conception, chlorhexidine has proven its effectiveness beyond dispute, and its different formulations are used routinely in both general dental practice and teaching institutions. Chlorhexidine gluconate had generated considerable interest in the dental community since its introduction as a $0.2 \%$ mouthrinse in an experimental gingivitis study [7].

Essential oils of various medicinal plants have been used as mouth rinses in reducing plaque, gingivitis and also as antibacterial agents. They received a positive endorsement from W.D. Miller as a "very useful and active antiseptic" against oral bacteria. Despite a plethora of germicidal claims for most of the twentieth century, the dental profession has 
not taken mouth rinses seriously [8].

Although numerous antiplaque, antigingivitis studies have been conducted with first generation agents, the largest body of work has been presented with essential oil mouthrinses. As a result of this, a large number of studies conducted in 1970's and 80's, the Council of Dental Therapeutics accepted essential oils as effective mouthrinse. Essential oil mouth rinses also enervated interest due to the lack of side effects associated with its prolonged use [7]. In the long term studies the plaque reduction varied from $14 \%$ to $34 \%$ and the reduction in gingivitis was $22 \%$ to $34 \%$ as compared to placebo. There were no mucosal aberrations or development of extrinsic stain reported in these studies. Some patients noted an initial burning sensation but accustomization usually occurred in a few days [9].

In general, the level of reduction in plaque and gingivitis seen with chlorhexidine is greater than that noted for the essential oil mouthrinses. This difference has been attributed to its substantivity [10]. This must be balanced against the disquieting characteristic of chlorhexidine to form a yellowish brown stain on teeth, tongue, plastic and composite restorations, and on artificial teeth. To counteract the side effects of chlorhexidine many other mouthrinses are launched in the market commercially. But their efficacy with comparison to chlorhexidine is not yet established [11].

\section{Effect of Dill Seed Oil on Gingivitis and Plaque Scores}

The gingival index was used to assess the levels of gingivitis in the study subjects at baseline, 45 days and 90 days and comparisons were made. In both the groups there was a significant reduction of gingival scores from baseline to 45 days and from 45 days to 90 days. When mean scores of dill seed oil was compared with chlorhexidine group, there was no statistically significant difference in gingival index scores at both 45 days $(p=0.69)$ and 90 days $(p=0.76)$. It shows that there is no significant difference in gingival scores from baseline to 45 and 90 days between dill seed oil and chlorhexidine group (Table 1).

When mean scores of dill seed oil was compared with chlorhexidine group, there was no statistically significant difference in plaque index scores at both 45 days $(p=0.62)$ and 90 days $(p=0.79)$. It shows that there is no significant difference in gingival scores from baseline to 45 and 90 days between essential oil and chlorhexidine group (Table 2 ).

It was observed that there is statistical difference in gingival and plaque scores when compared with baseline to 45 days $(p<0.001)$, baseline to 90 days $(p<0.001)$ and 45 days to 90 days $(p<0.001)$ when intragroup comparisons were done (Tables 3, 4).

The antiplaque activity of chlorhexidine may be attributed to three mechanisms for inhibition of plaque, i.e ; the effective blocking of acidic groups of salivary glycoproteins will reduce their adsorption to hydroxyapatite and formation of acquired pellicle; the ability of bacteria to bind to tooth surfaces may be reduced by adsorption of chlorhexidine to the extracellular polysaccharides of their capsules or gylcocalyces and the chlorhexidine may compete with calcium ions for acidic agglutination factors in plaque [10].

Chlorhexidine differs from the rest of antiplaque agents in its persistent, long lasting bacterostatic action, also termed as 'Substantivity'. The action lasts for as long as 12 hours in the oral cavity after a single rinse. The dicationic chlorhexidine molecule, attaches to the pellicle by one cation, and to the bacteria attempting to colonize the tooth surface with the other. This is called the 'Pin-Cushion Effect'. This prolongs the action of Chlorhexidine [10].

The present results indicate that the use of dill seed oil and $0.2 \%$ chlorhexidine gluconate mouthrinse has an advantage over the mechanical plaque removal methods alone. The majority of the population may not perform mechanical plaque removal sufficiently. Thus, antimicrobial mouthrinses that augment daily home care may provide an effective means of removing or controlling bacterial plaque to limit gingivitis and periodontitis [12, 13]. In fact, it has been shown that chemotherapeutic mouthrinses are effective measures for patients with gingivitis, providing a clinically significant and meaningful benefit in the reduction of plaque and gingivitis. Mouthrinses have the ability to deliver therapeutic ingredients and to benefit all accessible surfaces in the mouth including interproximal hard and soft tissues and, depending on their composition, remain active for extended periods. It was concluded that dill seed oil and Hexodent $(0.2 \%$ chlorhexidine gluconate $)$ mouthrinse have similar antiplaque and antigingival effectiveness.

\section{CONCLUSION}

It can be concluded that Dill seed oil mouthrinse and $0.2 \%$ chlorhexidine gluconate mouthrinse (Hexodent) have similar antiplaque and antigingival effectiveness. For short-term antiplaque effects, $0.2 \%$ chlorhexidine gluconate remains the "chemical toothbrush" of choice. Dill seed oil mouthrinse can be used as an alternative for short term 
maintenance therapy and has an advantage of having fewer side effects. As enough brands of dill seed oil mouthrinses are not available in the market, pharmaceutical companies should come up with better formulation, taste and compatibility to use, hence further research is needed in this direction.

The limitation of the present study is that it's a first of a kind wherein dill seed oil extract has been used as a mouth rinse to prove antigingival and antiplaque effects. Hence not many comparisons could be drawn. It is recommended that the dill seed oil mouthrinse has to be tested on large population and at community levels. Further the antibacterial properties of dill seed oil against oral bacteria has to be explored.

\section{CONFLICT OF INTEREST}

The authors confirm that this article content has no conflict of interest.

\section{ACKNOWLEDGEMENTS}

Declared none.

\section{REFERENCES}

[1] Fine DH, Markowitz K, Furgang D, et al. Effect of an essential oil-containing antimicrobial mouthrinse on specific plaque bacteria in vivo. J Clin Periodontol 2007; 34(8): 652-7.

[http://dx.doi.org/10.1111/j.1600-051X.2007.01096.x] [PMID: 17535287]

[2] Charles CH, Sharma NC, Galustians HJ, Qaqish J, McGuire JA, Vincent JW. Comparative efficacy of an antiseptic mouthrinse and an antiplaque/antigingivitis dentifrice. A six-month clinical trial. J Am Dent Assoc 2001; 132(5): 670-5. [http://dx.doi.org/10.14219/jada.archive.2001.0245] [PMID: 11367972]

[3] Overholser CD, Meiller TF, DePaola LG, Minah GE, Niehaus C. Comparative effects of 2 chemotherapeutic mouthrinses on the development of supragingival dental plaque and gingivitis. J Clin Periodontol 1990; 17(8): 575-9. [http://dx.doi.org/10.1111/j.1600-051X.1990.tb01108.x] [PMID: 2212088]

[4] Sharma NC, Charles CH, Qaqish JG, Galustians HJ, Zhao Q, Kumar LD. Comparative effectiveness of an essential oil mouthrinse and dental floss in controlling interproximal gingivitis and plaque. Am J Dent 2002; 15(6): 351-5.

[PMID: 12691269]

[5] Praveen D, Sharmishtha P. Phytochemical analysis and antibacterial efficacy of dill seed oil against multi-drug resistant clinical isolates. Asian J Pharmaceut Clin Res 2012; 5(2): 112-7.

[6] Brecx M. Strategies and agents in supragingival chemical plaque control. Periodontol 2000 1997; $15: 100-8$. [http://dx.doi.org/10.1111/j.1600-0757.1997.tb00109.x] [PMID: 9643237]

[7] Charles CH, Mostler KM, Bartels LL, Mankodi SM. Comparative antiplaque and antigingivitis effectiveness of a chlorhexidine and an essential oil mouthrinse: 6-month clinical trial. J Clin Periodontol 2004; 31(10): 878-84.

[http://dx.doi.org/10.1111/j.1600-051X.2004.00578.x] [PMID: 15367192]

[8] Moran JM. Chemical plaque control-prevention for the masses. Periodontol 2000 1997; 15: 109-17. [http://dx.doi.org/10.1111/j.1600-0757.1997.tb00110.x] [PMID: 9643238]

[9] Wu CD, Savitt ED. Evaluation of the safety and efficacy of over-the-counter oral hygiene products for the reduction and control of plaque and gingivitis. Periodontol 2000 2002; 28: 91-105.

[http://dx.doi.org/10.1034/j.1600-0757.2002.280105.x] [PMID: 12013351]

[10] Jones CG. Chlohexidine: is it still the gold standard? Periodontology 2000 1997; 28: 55-62. [PMID: 9643233]

[11] Southern EN, McCombs GB, Tolle SL, Marinak K. The comparative effects of $0.12 \%$ chlorhexidine and herbal oral rinse on dental plaqueinduced gingivitis. J Dent Hyg 2006; 80(1): 12. [PMID: 16451766]

[12] Patel RM, Malaki Z. The effect of a mouthrinse containing essential oils on dental plaque and gingivitis. Evid Based Dent 2008; 9(1): $18-9$. [http://dx.doi.org/10.1038/sj.ebd.6400566] [PMID: 18364689]

[13] Masline S, Close B. Aromatherapy: The A-Z Guide to Healing with Essential Oils. New York: Dell book Publisher 1997.

(C) Eshwar et al.; Licensee Bentham Open.

This is an open access article licensed under the terms of the Creative Commons Attribution-Non-Commercial 4.0 International Public License (CC BY-NC 4.0) (https://creativecommons.org/licenses/by-nc/4.0/legalcode), which permits unrestricted, non-commercial use, distribution and reproduction in any medium, provided the work is properly cited. 